\title{
LEXICOGRAPHIC-TYPE EXTENSION OF MIN-MAX LOGIC IS NOT UNIQUELY DETERMINED
}

\author{
Olga Kosheleva \\ Ph.D. (Phys.-Math.), Associate Professor, e-mail: olgak@utep.edu \\ Vladik Kreinovich \\ Ph.D. (Phys.-Math.), Professor, e-mail: vladik@utep.edu \\ University of Texas at El Paso, El Paso, Texas 79968, USA
}

\begin{abstract}
Since in a computer, "true" is usually represented as 1 and "false" as 0 , it is natural to represent intermediate degrees of confidence by numbers intermediate between 0 and 1 ; this is one of the main ideas behind fuzzy logic - a technique that has led to many useful applications. In many such applications, the degree of confidence in $A \& B$ is estimated as the minimum of the degrees of confidence corresponding to $A$ and $B$, and the degree of confidence in $A \vee B$ is estimated as the maximum; for example, $0.5 \vee 0.3=0.5$. It is intuitively $\mathrm{OK}$ that, e.g., $0.5 \vee 0.3<0.51$ and, more generally, that $0.5 \vee 0.3<0.5+\varepsilon$ for all $\varepsilon>0$. However, intuitively, an additional argument in favor of the statement should increase our degree of confidence, i.e., we should have $0.5<0.5 \vee 0.3$. To capture this intuitive idea, we need to extend the min-max logic from the interval $[0,1]$ to a lexicographic-type order on a larger set. Such extension has been proposed - and successfully used in applications - for some propositional formulas. A natural question is: can this construction be uniquely extended to all "and"-"or" formulas? In this paper, we show that, in general, such an extension is not unique.
\end{abstract}

Keywords: fuzzy logic, min-max logic, lexicographic extension.

\section{Formulation of the Problem}

Need for intermediate degrees of belief. In the usual 2-valued logic, every statement is either true or false. In a computer, "true" is usually represented as 1 , and "false" as 0.

In practice, for many statements, we do not know whether they are true or false, but we have some degree of confidence that they are true. A reasonable idea is to describe this degree of confidence by numbers intermediate between 0 (false, absolutely no confidence) and 1 (true, absolute confidence). Using such degrees of confidence is one of the main ideas behind fuzzy logic, a technique that has been successful in many applications; see, e.g., [5, 8-12,15].

Need for "and"- and "or"-operations. For each statement provided by an expert, we can ask this expert to also provide his/her degree of confidence in this 
statement. However, to make conclusions, we usually need to use several such statements.

For example, sometimes, the conclusion is true only if both statements $A$ and $B$ are true, i.e., if a composite statement $A \& B$ is true. Sometime, the conclusion can be derived from each of these statements, so the conclusion is true if either $A$ is true or $B$ is true, i.e., if a composite statement $A \vee B$ is true. In general, we can have more complex composite statements.

So, in addition to degrees of confidence in individual statements, we also need to know degrees of confidence in such composite statements. For $n$ basic statements $A_{1}, \ldots, A_{n}$, we can have exponentially many composite statements - e.g., we have $A_{i_{1}} \& \ldots \& A_{i_{k}}$ for each of $2^{n}-(n+1)$ non-trivial subsets $\left\{i_{1}, \ldots, i_{k}\right\} \subseteq\{1, \ldots, n\}$. Even for reasonable-size $n$ like $n=30$, this means billions of possible composite statements. There is no way we can ask the experts to provide degree of confidence in each of these statements. So, we need to be able to estimate the degree of confidence in such statements - in particular, in statements $A \& B$ and $A \vee B-$ based on the known degrees of confidence $a$ and $b$ in statements $A$ and $B$. For "and"- and "or"-statements, the resulting estimates are known as "and"-operations and "or"-operations, or, for historical reasons, t-norms and t-conorms. In this paper, we will denote these operations by $a \& b$ and $a \vee b$.

These operations much satisfy some natural requirements. For example, since $A \& B$ means the same as $B \& A$, it is reasonable to require these two formulas should result in the same estimate, i.e., that we should always have $a \& b=b \& a$. Similarly, the fact that $A \&(B \& C)$ means the same as $(A \& B) \& C$ implies that it is reasonable to have $a \&(b \& c)=(a \& b) \& c$, etc.

Our degree of confidence in a stronger statement " $A$ and $B$ " cannot be larger than our degree of confidence in each individual statement, i.e., we must have $a \& b \leqslant a$ and $a \&, b \leqslant b$. Similarly, our degree of confidence in a weaker statement $A \vee B$ cannot be smaller than our degree of confidence in each of the original statements, so we should have $a \leqslant a \vee b$ and $b \leqslant a \vee b$.

There many other similar natural requirements. There are many different "and"and "or"-operations satisfying all these requirements; see, e.g., [5, 8-12, 15].

Min-max logic. It seems natural to also impose some additional requirements: e.g., if our degree of confidence in a statement $C$ is larger than (or equal to) our degrees of confidence in $A$ and in $B$, then it should also be larger than or equal to our degree of confidence in a statement " $A$ or $B$ ". In precise terms: if $a \leqslant c$ and $b \leqslant c$, then we should have $a \vee b \leqslant c$.

For $a \leqslant b$ and $c=b$, the fact that we have $a \leqslant b$ and $b \leqslant b$ immediately implies that $a \vee b \leqslant b$. Since we always have $b \leqslant a \vee b$, this implies that $a \vee b=\max (a, b)$, i.e., that we have a max "or"-operation.

Similar, it is reasonable to require that if $c \leqslant a$ and $c \leqslant b$, then $c \leqslant a \& b$. For $a \leqslant b$ and $c=a$, since we have $a \leqslant a$ and $a \leqslant b$, we thus imply that $a \leqslant a \& b$. Since we always have $a \& b \leqslant a$, this implies that $a \& b=\min (a, b)$, i.e., that we have a min "and"-operations.

Need for a lexicographic extension. Formally, in the min-max logic, we have, 
e.g., $0.5 \vee 0.3=0.5$. However, intuitively, if we have an additional argument in favor of the statement - even if that additional argument is weaker than the original one - this should boost our degree of confidence in a statement.

In other words, it is OK that $0.5 \vee 0.3$ is smaller than 0.6 , smaller than 0.51 , smaller than 0.501 - and, in general, smaller than $0.5+\varepsilon$ for an arbitrary small $\varepsilon>0$, but we would like to require that $0.5<0.5 \vee 0.3$. So, it is desirable to extend the set of possible values of degree of confidence from the interval $[0,1]$ to some more general ordered set.

A possibility to have values which are greater than 0.5 but smaller than all larger numbers occurs, e.g., in lexicographic orderings of pairs of non-negative numbers, when $\left(a_{1}, a_{2}\right)<\left(b_{1}, b_{2}\right)$ if and only if:

- either $a_{1}<b_{1}$

- or $a_{1}=b_{1}$ and $b_{1}<b_{2}$;

in this case, $(0.5,0)<(0.5,0.3)$ but $(0.5,0.3)<(0.5+\varepsilon, 0)$ for all $\varepsilon>0$. So, it is reasonable to call the desired extension lexicographic-type.

For some composite formulas, such an extension was proposed and used in $[1-4,14]$. This extension was successfully use to deal with uncertainty in petroleum engineering and in other application areas; see, e.g., [14].

Comment. What we should get is, in effect, a new value which differs from 0.5 by an infinitesimal number - similar to what is done in nonstandard analysis; see, e.g., $[6,7,13]$.

Natural question. A natural question is: how unique is this extension?

Our conclusion is that it is not unique.

\section{Our Answer}

A natural formalization. Let us first formulate the above question in precise terms.

We want to consider expressions $E$ of the type $a, a \vee b, a \&(b \vee c)$, i.e., expressions obtained from numbers from the interval $[0,1]$ by using symbols $\vee$ and $\&$.

The following natural formalization comes from fact that for most other "or"-operations, we have $a<a \vee b$ for all $a<1$. The max-operation can be represented as a limit of such operations. Similarly, for most other "and"-operations, we have $a \& b<a$ for all $a>0$. The min-operation can be represented as a limit of such operations.

So, let us consider a family $\vee_{p}$ of "or"-operations:

- that tend to $\max (a, b)$, i.e., for which $a \vee_{p} b \rightarrow \max (a, b)$ as $p \rightarrow \infty$, and

- for which, for each $a<1$ and $b$, we have $a<a \vee_{p} b$ for all sufficiently large $p$. 
We can have many such families. For example, we can take

$$
a \vee_{p} b=\min \left(\left(a^{p}+b^{p}\right)^{1 / p}, 1\right) .
$$

One can easily check that all the elements of this sequence are "or"-operations ( $t$ conorms), and that the above expression indeed tends to $\max (a, b)$ as $p$ increases.

Similarly, let us consider a family $\&_{p}$ of "and"-operations that:

- tend to $\min (a, b)$, i.e., for which $a \&_{p} b \rightarrow \min (a, b)$ as $p \rightarrow \infty$, and

- for which, for all $a>0$ and $b$, we have $a \&_{p} b<a$ for all sufficiently large $p$.

We can have many such families. For example, we can take

$$
a \&_{p} b=\left(a^{-k \cdot p}+b^{-k \cdot p}\right)^{-1 /(k \cdot p)},
$$

for some $k>0$. One can easily check that all the elements of this sequence are "and"-operations (t-norms), and that the above expression indeed tends to $\min (a, b)$ as $p$ increases.

For each expression and for each $p$, we can get a value $E_{p}$ if we interpret $\vee$ as $\vee_{p}$ and $\&$ as $\&_{p}$. For example, for the expression $E=0.3 \vee(0.5 \& 0.4)$, we have $E_{p} \stackrel{\text { def }}{=} 0.3 \vee_{p}\left(0.5 \&_{p} 0.4\right)$. In the limit $p \rightarrow \infty$, the value $E_{p}$ tends to the value of $E$ in the min-max logic.

For two expressions $E$ and $E^{\prime}$, we can then say that $E<E^{\prime}$ if for all sufficiently large $p$, we have $E_{p}<E_{p}^{\prime}$. By the properties of the operations $\vee_{p}$, this will guarantee, e.g., that $0.5<0.5 \vee 0.3$, and, in general, that $a<a \vee b$ for all $a<1$ and $b$.

Now, we can formulate the above question in precise terms.

Question. When $a<1$, then for expressions $a$ and $a \vee b$, we have $a<a \vee b$ no matter what families $\vee_{p}$ and $\&_{p}$ we select. In this sense, for these two expressions, the lexicographic-type extension of min-max logic is unique.

A natural question is whether this is true for all pairs of expressions.

Our answer. Our answer is that there exist pairs of expressions $E$ and $E^{\prime}$ for which the order depends on which families $\vee_{p}$ and \& $\&_{p}$ we select: for some families, we have $E<E^{\prime}$, while for others, we have $E^{\prime}<E$.

As an example, we can take the expressions $E=(a \& a) \vee(a \& a)$ and $E^{\prime}=a$ for which $E_{p}=\left(a \&_{p} a\right) \vee_{p}\left(a \&_{p} a\right)$ and $E_{p}^{\prime}=a$.

Different orders can be observed already for the above examples of families: specifically, different orders can be observed for different values $k$.

When $k$ is very large, then, in comparison with the "or"-operation, we practically have $a \&_{p} b \approx \min (a, b)$. In particular, $a \&_{p} a \approx a$, and thus, the value $E_{p}$ is thus approximately equal to $a \vee_{p} a$. We know that $a<a \vee_{p} a$, so in this case, we have $E_{p}^{\prime}<E_{p}$, and thus, by definition $E^{\prime}<E$.

On the other hand, when $k$ is very small, then, in effect, the opposite happens: in comparison with the "and"-operation, we practically have $a \vee_{p} b \approx \max (a, b)$. Thus,

$$
E_{p}=\left(a \&_{p} a\right) \vee_{p}\left(a \&_{p} a\right) \approx \max \left(a \&_{p} a, a \&_{p} a\right)=a \&_{p} a .
$$


We know that $a \&_{p} a<a$, so in this case, we have $E_{p}<E_{p}^{\prime}$ and thus, $E<E^{\prime}$.

Non-uniqueness is proven.

\section{Acknowledgments}

This work was supported in part by the National Science Foundation grants 1623190 (A Model of Change for Preparing a New Generation for Professional Practice in Computer Science) and HRD-1242122 (Cyber-ShARE Center of Excellence).

The authors are thankful to Ildar Batyrshin for valuable discussions.

\section{REFERENCES}

1. Batyrshin I.Z. Lexicographic estimates of the likelihood with universal bounds. I. Transcations of the Russian Academy of Sciences: Technical Cybernetics, 1994, no. 5, pp. $44-59$.

2. Batyrshin I.Z. Modus ponens generating function in the class of $\Lambda$-valuations of plausibility. Proceedings of the Tenth Conference on Uncertainty in Artificial Intelligence, Seattle, Washington, July 29-31, 1994, pp. 55-59.

3. Batyrshin I.Z. Uncertainties with memory in construction of strict monotonic t-norms and t-conorms for finite ordinal scales: basic definitions and applications. Applied and Computational Mathematics, 2011, vol. 10, no. 3, pp. 498-513.

4. Batyrshin I.Z. and Batyrshin I.I. Negations, strict monotonic t-norms and t-conorms for finite ordinal scales. Proceedings of the 8th Joint Conference on Information Sciences (JCIS) and 10th International Conference on Fuzzy Theory and Technology, Salt Lake City, July 21-26, 2005, pp. 50-53.

5. Belohlavek R., Dauben J.W., and Klir G.J. Fuzzy Logic and Mathematics: A Historical Perspective. Oxford University Press, New York, 2017.

6. Goldblatt R. Lectures on the Hyperreals: An Introduction to Nonstandard Analysis. Springer, Berlin, Heidelberg, New York, 1998.

7. Gordon E.I., Kusraev A.G. and Kutateladze S.S. Infinitesimal Analysis. Kluwer, Dordrecht, 2002.

8. Klir G. and Yuan B. Fuzzy Sets and Fuzzy Logic. Prentice Hall, Upper Saddle River, New Jersey, 1995.

9. Mendel J.M. Uncertain Rule-Based Fuzzy Systems: Introduction and New Directions. Springer, Cham, Switzerland, 2017.

10. Nguyen H.T. and Kreinovich V. Nested intervals and sets: concepts, relations to fuzzy sets, and applications. In: R.B. Kearfott and V. Kreinovich (eds.). Applications of Interval Computations, Kluwer, Dordrecht, 1996, pp. 245-290.

11. Nguyen H.T., Walker C., and Walker E.A. A First Course in Fuzzy Logic. Chapman and Hall/CRC, Boca Raton, Florida, 2019.

12. Novák V., Perfilieva I., and Močkoř J. Mathematical Principles of Fuzzy Logic. Kluwer, Boston, Dordrecht, 1999.

13. Robinson A. Nonstandard Analysis. Princeton University Press, Princeton, New Jersey, 1996. 
14. Sheremetov L., Batyrshin I.Z., Filatov D., Martinez J., and Rodriguez H. Fuzzy expert system for solving lost circulation problem. Applied Soft Computing, 2008, vol. 8, pp. 14-29.

15. Zadeh L.A. Fuzzy sets. Information and Control, 1965, vol. 8, pp. 338-353.

\title{
РАСШИРЕНИЕ ЛЕКСИКОГРАФИЧЕСКОГО ТИПА ЛОГИКИ МИНИМАКСА НЕ ОПРЕДЕЛЕНО ОДНОЗНАЧНО
}

\author{
О. Кошелева \\ к.ф.-м.н., доцент, е-mail: olgak@utep.edu \\ В. Крейнович \\ к.ф.-м.н., профессор, e-mail: vladik@utep.edu \\ Техасский университет в Эль Пасо, США
}

\begin{abstract}
Аннотация. Поскольку в компьютере «истина» обычно представлена как 1, а «ложь»- как 0 , естественно представлять промежуточные степени достоверности числами между 0 и 1 ; это одна из основных идей, лежащих в основе it нечёткой логики - техники, которая привела к множеству полезных приложений. Во многих таких приложениях степень уверенности в $A \& B$ оценивается как минимум из степеней уверенности, соответствующих $A$ и $B$, а степень уверенности в $A \vee B$ оценивается как максимум; например $0,5 \vee 0,3=0,5$. Интуитивно понятно, что, например, $0,5 \vee 0,3<0,51$ и, в более общем смысле, $0,5 \vee 0,3<0,5+\varepsilon$ для всех varepsilon $>0$. Однако интуитивно дополнительный аргумент в пользу утверждения должен повысить нашу степень уверенности, т.е. мы должны иметь $0,5<0,5 \vee 0,3$. Чтобы уловить эту интуитивную идею, нам нужно расширить логику минимакса с интервала $[0,1]$ до порядка лексикографического типа на большем множестве. Такое расширение было предложено - и успешно используется в приложениях - для некоторых формул высказываний. Возникает естественный вопрос: можно ли однозначно распространить эту конструкцию на все формулы «и» - «или»? В этой статье мы показываем, что такое расширение, вообще говоря, не единственно.
\end{abstract}

Ключевые слова: нечеткая логика, минимаксная логика, лексикографическое расширение.

Дата поступления в редакцию: 13.07.2020 